\title{
Las Campanas Loose Groups in the supercluster-void network
}

\author{
M. Einasto ${ }^{1}$, J. Jaaniste ${ }^{1,2}$, J. Einasto $^{1}$, P. Heinämäki ${ }^{1,3}$, V. Müller ${ }^{4}$, and D. L. Tucker ${ }^{5}$ \\ 1 Tartu Observatory, 61602 Tõravere, Estonia \\ 2 Institute of Physics, Estonian Agricultural University, Kreutzwaldi 64, 51014 Tartu, Estonia \\ 3 Tuorla Observatory, Väisäläntie 20, Piikkiö, Finland \\ 4 Astrophysical Institute Potsdam, An der Sternwarte 16, 14482 Potsdam, Germany \\ 5 Fermi National Accelerator Laboratory, MS 127, PO Box 500, Batavia, IL 60510, USA
}

Received 23 January 2003 / Accepted 17 April 2003

\begin{abstract}
We study the spatial distribution of loose groups from the Las Campanas Redshift Survey, comparing it with the supercluster-void network delineated by rich clusters of galaxies. We use density fields and the friends-of-friends (FoF) algorithm to identify the members of superclusters of Abell clusters among the Las Campanas loose groups. We find that systems of loose groups tend to be oriented perpendicularly to the line-of-sight, and discuss possible reasons for that. We show that loose groups in richer systems (superclusters of Abell clusters) are themselves also richer and more massive than groups in systems without Abell clusters. Our results indicate that superclusters, as high density environments, have a major role in the formation and evolution of galaxy systems.
\end{abstract}

Key words. cosmology: observations - cosmology: large-scale structure of the Universe

\section{Introduction}

The first evidence that the large scale structure of the Universe forms a web-like network of galaxy systems was obtained in the 1970's (Jõeveer \& Einasto 1978; Tarenghi et al. 1978). In the supercluster-void network, superclusters of galaxies with characteristic dimensions of up to $100 \mathrm{~h}^{-1} \mathrm{Mpc}^{1}$ are the largest relatively isolated density enhancements in the Universe. As commonly accepted, superclusters are formed by perturbations of the same wavelength in the initial density field (Einasto et al. 2001 and references therein).

Superclusters have mainly been studied using the data on rich clusters of galaxies (Einasto et al. 2001, and references therein). Properties of superclusters (their shapes and orientations) have been studied by West (1989), Plionis et al. (1992), Jaaniste et al. (1998) and Kolokotronis et al. (2002). Already early studies of the fine structure of nearby superclusters and of the distribution of matter in low density regions between superclusters (Lindner et al. 1995 and references therein) showed that superclusters have a complicated structure, where clusters and groups of galaxies are connected by filaments of galaxies. Superclusters may also contain hot gas (Kull \& Böhringer 1999; Bardelli et al. 2000; Rines et al. 2001; Rose et al. 2002).

At present several deep galaxy surveys are publicly available. Among these surveys are the ESO Slice Project survey (ESP, Vettolani et al. 1997), the Las Campanas Redshift Survey (LCRS; Shectman et al. 1996), the 2 degree Field Galaxy

Send offprint requests to: M. Einasto, e-mail: maret@aai.ee

${ }^{1} h$ is the Hubble constant in units of $100 \mathrm{~km} \mathrm{~s}^{-1} \mathrm{Mpc}^{-1}$.
Redshift Survey (2dF, Colless et al. 2001) and the Sloan Digital Sky Survey (SDSS, York et al. 2000). These surveys can be used to study the structure of a large number of superclusters in more detail and on larger scales than hitherto possible.

The catalogue of loose groups of galaxies extracted from the LCRS (LCLGs, Tucker et al. 2000, hereafter TUC) gives us an opportunity to study the spatial distribution and intrinsic properties of loose groups on large scales, up to redshifts $z \approx 0.15$. In the LCRS, galaxies have been observed in 6 thin slices; thus, in order to use this survey to study the 3D structure of the Universe, it is necessary to analyse this survey together with data on rich clusters of galaxies, e.g. Abell clusters. Such a combined analysis of the spatial distribution of loose groups, Abell clusters, and superclusters of Abell clusters enables us to exploit the deep slices to study the fine structure of superclusters and the hierarchy of the structures in the Universe.

In the present paper we found populations of LCLGs in superclusters of Abell clusters, using density fields and the friends-of-friends (FoF) analysis of the LCRS. We studied the properties of these systems and the distribution of LCLGs with respect to the supercluster-void network, traced by Abell clusters. We also studied the properties of LCLGs in systems that contain no Abell clusters.

In the next section we describe our LCLG and Abell cluster samples. In Sect. 3 we identify LCLGs that belong to superclusters. Then we study the properties of superclusters and the distribution of LCLGs with respect to the supercluster-void network. In the last two sections we give a discussion and summary of our results. 


\section{Observational data}

\subsection{LCRS loose groups}

The LCRS (Shectman et al. 1996) is an optically selected galaxy redshift survey that extends to a redshift of 0.2 and is composed of six slices, each covering an area of roughly $1.5^{\circ} \times 80^{\circ}$. Three of these slices are located in the Northern Galactic Cap and are centred at the declinations $\delta=-3^{\circ},-6^{\circ},-12^{\circ}$; the other three slices are located in the Southern Galactic Cap and are centred at the declinations $\delta=-39^{\circ},-42^{\circ},-45^{\circ}$. The thickness of slices is approximately $7.5 h^{-1} \mathrm{Mpc}$ at the survey's median redshift. Altogether, the LCRS contains redshifts for 23697 galaxies within its official photometric and geometric boundaries.

The survey spectroscopy was carried out using a 50 fibre multiobject spectrograph with the nominal apparent magnitude limits for the spectroscopic fields $16 \leq R \leq 17.3$, and a 112 fibre multiobject spectrograph with a larger range of apparent magnitudes $(15 \leq R \leq 17.7)$. Therefore, the selection criteria varied from field to field, often within a given slice.

Using the FoF percolation algorithm, TUC extracted the LCLG catalogue from the LCRS. The linking lengths were chosen so that each group is contained within a galaxy number density enhancement contour of $\delta n / n=80$. When extracting these LCLGs, great care was taken to account for the radial selection function, the field-to-field selection effects inherent in the LCRS, and the boundary effects due to the fact that the LCRS is composed of six thin slices. As the derived properties of the LCLGs in the 50-fibre fields do not differ substantially from the derived properties of the LCLGs in the 112-fibre fields, the selection effects were successfully eliminated.

The LCLG catalogue contains 1495 groups in the redshift range of $10000 \leq c z \leq 45000 \mathrm{~km} \mathrm{~s}^{-1}$. This is one of the first deep, wide samples of loose groups; as such, it enables us for the first time to investigate the spatial distribution and properties of groups in a large volume.

\subsection{Abell clusters and superclusters}

We use Andernach \& Tago's (1998) recent compilation of data on rich clusters of galaxies (Abell 1958 and Abell et al. 1989). From this compilation we selected clusters of all richness classes out to the redshift $z=0.13$; we, however, excluded clusters from the generally poorer supplementary (or " $S$ ") catalogue of clusters compiled by Abell et al. (1989). Our final Abell cluster sample contains 1665 clusters, of which 1071 have measured redshifts for two or more galaxies. Using this sample of Abell clusters Einasto et al. (2001) compiled a catalogue of superclusters of Abell clusters ("Abell superclusters") and a list of X-ray clusters in superclusters. In the present paper we use this catalogue as a reference to study supercluster membership of LCLGs. The X-ray data were also taken from the lists by Schwope et al. (2000, hereafter RBS) and by de Grandi et al. (1999). Information on radio clusters has been taken from Ledlow \& Owen (1995).

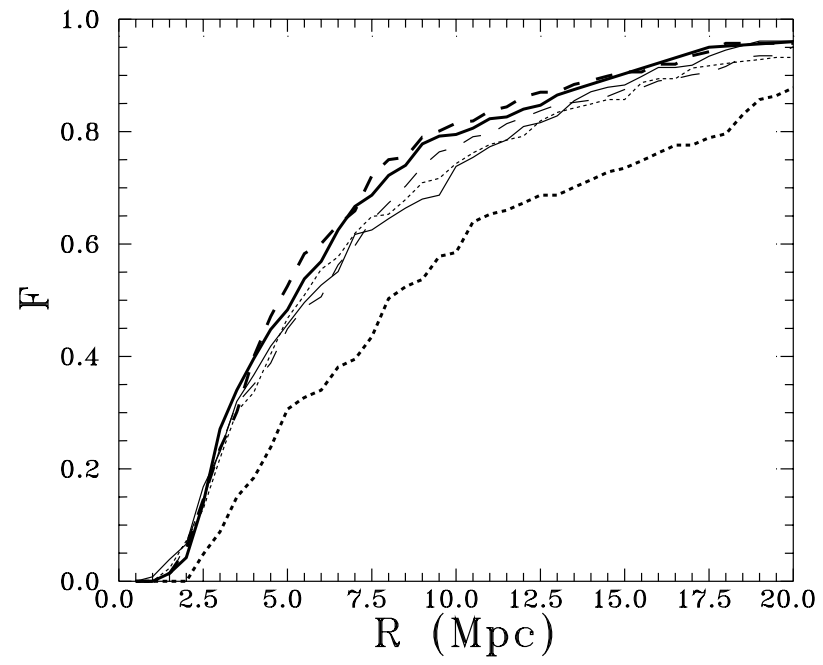

Fig. 1. Multiplicity function of LCLGs showing the fraction of groups in systems with at least 2 members as a function of the neighbourhood radius. Bold lines: solid line - slice $\delta=-3^{\circ}$, dotted line slice $\delta=-6^{\circ}$, dashed line - slice $\delta=-12^{\circ}$. Thin lines: solid line: slice $\delta=-39^{\circ}$, dotted line - slice $\delta=-42^{\circ}$, and dashed line - slice $\delta=-45^{\circ}$. Note the difference between the slice $\delta=-6^{\circ}$ and other slices.

\section{Las Campanas Loose Groups and superclusters}

Einasto et al. (2001) identified superclusters of Abell clusters ("Abell superclusters") using the FoF algorithm with linking the length $R=24 h^{-1} \mathrm{Mpc}$ that corresponds approximately to an overdensity contour of 2 . This radius was chosen by studying the spatial distribution of rich clusters of galaxies. This list helps us to identify Abell superclusters that cross the LCRS slices. Using the same linking length to search for members of superclusters among the loose groups links almost 90-95\% of groups (Fig. 1). At the other extreme, by simply identifying as supercluster members those loose groups that are located around rich clusters in a sphere of radius $R=6 h^{-1} \mathrm{Mpc}$, as done by Einasto et al. (2003a), we miss the outer members of superclusters. Thus we have used additional methods to determine the system membership. We identified systems of loose groups and then searched for those systems that belong to Abell superclusters as described in the following two subsections.

\subsection{The FoF approach}

To identify systems of loose groups we applied the FoF algorithm to LCLGs from the each Las Campanas slice separately using a wide range of linking lengths (or neighbourhood radii). Figure 1 shows the fraction of LCLGs in systems with at least 2 member groups as a function of the neighbourhood radius. This figure shows that at a neighbourhood radius $R=6 h^{-1} \mathrm{Mpc}, 50 \%-60 \%$ of groups belong to such systems. We chose this radius, $R=6 h^{-1} \mathrm{Mpc}$, as our linking length to identify systems of LCLGs. The same radius was used to search for populations of loose groups around rich clusters in Einasto et al. (2003a). A more detailed analysis of the multiplicity functions shows that at this neighbourhood radius richer systems 
(with at least 8 member groups) start to form. This was one of the reasons to use the neighbourhood radius $R=6 h^{-1} \mathrm{Mpc}$.

Figure 1 shows that the multiplicity function of groups from the $\delta=-6^{\circ}$ slice differs from the multiplicity functions for groups in other slices. This may be due to the selection effects, as in the case of this slice all-but-two fields were observed with the 50-fibre spectrograph, and the selection effects that may decrease the number of groups are stronger. Another possibility is that this slice crosses a region dominated by voids and thus the number of groups in this slice and the number of superclusters crossed by this slice is smaller (see also Table 1). To check the first possibility we recalculated the multiplicity functions, using the neighbourhood radius $R$ in units of the dimensionless radius, $r=R / R_{0}$, where $R_{0}=[3 V /(4 \pi N)]^{1 / 3}$ is the Poisson radius (the radius of a sphere which contains one particle), $N$ is the number of particles in the sample, and $V$ is the volume of the sample. The multiplicity function of the slice $\delta=-6^{\circ}$ still differed from the multiplicity functions of other slices. Thus this difference may be at least partly caused by peculiarities of the large scale distribution of groups in this slice.

Using the FoF technique, we occasionally find that some LCLGs, which appear to be associated with an Abell supercluster remain isolated even at the linking length $R=6 h^{-1} \mathrm{Mpc}$. Thus, to link these quasi isolated LCLGs to the supercluster, we could use either a variable linking length which would link these LCLGs to the supercluster system, or we could use another approach to determine system membership.

\subsection{The density field approach}

As another method to identify systems of loose groups and to determine membership of LCLGs within Abell superclusters we shall use the density field of the Las Campanas Survey slices calculated from the LCRS galaxy distribution. Details of these calculations will be published elsewhere (Einasto et al. 2003c). Here we shall only briefly outline the method.

To calculate the density field we formed a grid of cell size $1 h^{-1} \mathrm{Mpc}$ and used Gaussian smoothing (Einasto et al. 2003b). The thickness of the LCRS slices is only $1.5^{\circ}$; thus we calculated 2-dimensional density fields. To take into account the thickness of the slice, the smoothed density field was divided by the thickness of the slice at the location of a particular cell in real 3D space. In this way the map of the density field is reduced to that of a planar sheet of constant thickness.

To identify superclusters of galaxies we used the smoothing length $\sigma_{\mathrm{sm}}=10 \mathrm{~h}^{-1} \mathrm{Mpc}$. Numerical simulations have shown that this smoothing length is suitable for selecting supercluster-size density enhancements (Frisch et al. 1995; see also Basilakos et al. 2001).

The number of superclusters has a maximum for all slices at the relative threshold density $\Delta_{0}=1.5$ (Einasto et al. 2003b). The relative density $\Delta$ is expressed in units of the mean density $(\Delta \equiv \rho /\langle\rho\rangle)$, averaged over the whole observed area covered by a particular slice. For lower $\Delta_{0}$ superclusters merge; for higher $\Delta_{0}$ fewer high-density regions are counted. Our analysis shows that superclusters are still separated at limiting densities around
$\Delta_{0}=2.0$. This value, $\Delta_{0}=2.0$, defines compact and rather rich superclusters.

The density contrast in the large scale environment of superclusters (in the regions around Abell clusters that belong to superclusters) varies around $\Delta_{0}=2.0$. Therefore, in order to identify populations of loose groups that belong to superclusters, we use a variable threshold density limit to determine superclusters. In addition, we use the value of the overdensity in superclusters as one of the quantitative characteristics of the systems (Table 1).

In Table 1 we list superclusters, which intersect LCRS slices. In total we find 19 systems, 16 of which belong to Abell superclusters and 3 of which are relatively isolated. We denote the sample of loose groups in Abell superclusters as LCLG.scl, and the sample of loose groups in high-density systems without Abell clusters (LG superclusters) as LCLG.lgs.

The colour figures and the three-dimensional distribution of LCLGs, rich clusters and superclusters can be seen at the home page of Tartu Observatory (http://www.aai.ee/ maret/cosmoweb.html) and via EDP Sciences (http://www. edpsciences.org).

\section{Systems of Las Campanas Loose Groups}

\subsection{Approximation by the ellipsoid of concentration}

Superclusters (Einasto et al. 2001) are not regular systems with well-defined boundaries but aggregates of quite sparsely distributed clusters, groups and galaxies with some central concentration. To find the boundaries of such superclusters and to study their shape and orientation we approximate the spatial distribution of objects (clusters, groups, galaxies) in superclusters by a 3-dimensional ellipsoid of concentration. For such an ellipsoid we can find the centre, volume and principal axes. Although in most cases our superclusters do not form a regular body, these parameters help us to describe the density and alignments of the elements of large-scale structure.

In the present study we use the classical mass ellipsoid (see e.g. Korn \& Korn 1961):

$\sum_{i, j=1}^{3}\left(\lambda_{i j}\right)^{-1} x_{i} x_{j}=5$

where

$\lambda_{i j}=\frac{1}{N_{\mathrm{cl}}} \sum_{l=1}^{N_{c l}}\left(x_{i}^{l}-\xi_{i}\right)\left(x_{j}^{l}-\xi_{j}\right)$,

is the inertia tensor for equally weighted groups, $N_{\mathrm{cl}}$ is the total number of groups, and $\xi_{i}=\frac{1}{N_{\mathrm{cl}}} \sum_{l=1}^{N_{\mathrm{cl}}} x_{i}^{l}$ determines the Cartesian coordinates of the centre of mass of the system.

The formula determines a 3-dimensional ellipsoidal surface with the distance from the centre of the ellipsoid equal to the rms deviation of individual objects in the corresponding direction. This method can be applied for superclusters with $N \geq 5$. The problems related to the stability of the method and the influence of observational errors have been discussed in Jaaniste et al. (1998). 
Table 1. The data about loose groups in superclusters of Abell clusters and in rich systems without Abell clusters.

\begin{tabular}{|c|c|c|c|c|c|c|c|c|c|c|}
\hline $\begin{array}{r}N_{\mathrm{SCL}} \\
(1)\end{array}$ & $\begin{array}{l}\text { RA } \\
\text { (2) }\end{array}$ & $\begin{array}{r}D \\
(3)\end{array}$ & $\begin{array}{r}N_{\text {Abell }} \\
\text { (4) }\end{array}$ & $\begin{array}{r}N_{\mathrm{ALC}} \\
(5)\end{array}$ & $\begin{array}{l}N_{\mathrm{X}} \\
(6)\end{array}$ & $\begin{array}{r}N_{\text {LCLG }} \\
\text { (7) }\end{array}$ & $\begin{array}{r}\Delta \\
(8)\end{array}$ & $\begin{array}{r}a \\
(9)\end{array}$ & $\begin{array}{c}b / a \\
(10)\end{array}$ & $\begin{array}{c}\text { (ar) } \\
\text { (11) }\end{array}$ \\
\hline Slice & $\delta=$ & $-3^{\circ}$ & & & & & & & & \\
\hline 88 & 10.3 & 175 & 5 & - & & 23 & 3.5 & 22.9 & 0.49 & 79 \\
\hline 98 & 11.5 & 180 & 3 & 1 & & 6 & 1.3 & 11.1 & 0.18 & 84 \\
\hline 100 & 11.3 & 280 & 9 & 3 & & 23 & 2.6 & 68.7 & 0.45 & 12 \\
\hline 126 & 12.9 & 240 & 7 & 4 & 3 & 7 & 5.8 & 26.6 & 0.32 & 88 \\
\hline 155 & 15.2 & 280 & 2 & 1 & & 3 & 1.5 & & & \\
\hline $\operatorname{slg} 1$ & 14.6 & 170 & & & & 8 & 2.2 & 10.2 & 0.32 & 67 \\
\hline Slice & $\delta=$ & $-6^{\circ}$ & & & & & & & & \\
\hline 88 & 10.1 & 160 & 5 & 1 & & 6 & 4.4 & 9.2 & 0.29 & 64 \\
\hline 100 & 11.3 & 281 & 9 & 1 & & 3 & 1.6 & & & \\
\hline 267 & 11.9 & 348 & 4 & 1 & & 4 & 4.1 & & & \\
\hline Slice & $\delta=$ & $-12^{\circ}$ & & & & & & & & \\
\hline 88 & 10.2 & 169 & 5 & 1 & 1 & 2 & 0.9 & & & \\
\hline 119 & 12.6 & 272 & 6 & 1 & 1 & 15 & 4.3 & 21.0 & 0.20 & 87 \\
\hline 141 & 13.9 & 198 & 4 & 1 & 1 & 7 & 2.7 & 12.9 & 0.38 & 62 \\
\hline 156 & 15.1 & 308 & 2 & 1 & & 10 & 2.5 & 27.1 & 0.36 & 40 \\
\hline 274 & 13.8 & 248 & 2 & 1 & & 3 & 3.0 & & & \\
\hline $\operatorname{slg} 2$ & 11.6 & 219 & & & & 8 & 3.3 & 9.3 & 0.86 & 31 \\
\hline Slice & $\delta=$ & $-39^{\circ}$ & & & & & & & & \\
\hline 5 & 0.3 & 328 & 5 & 2 & & 4 & 2.2 & & & \\
\hline 8 & 0.6 & 182 & 3 & 1 & & 2 & 2.6 & & & \\
\hline 9 & 23.5 & 275 & 25 & 4 & & 14 & 2.4 & 49.6 & 0.41 & 53 \\
\hline 22 & 0.9 & 335 & 6 & 1 & & 2 & 1.0 & & & \\
\hline 23 & 1.1 & 216 & 2 & 2 & & 11 & 1.8 & 26.4 & 0.33 & 72 \\
\hline 37 & 2.0 & 287 & 5 & 1 & & 1 & 1.6 & & & \\
\hline 48 & 3.4 & 180 & 35 & 4 & & 24 & 4.2 & 47.5 & 0.58 & 21 \\
\hline 220 & 23.9 & 144 & 3 & 1 & & 3 & 1.7 & & & \\
\hline Slice & $\delta=$ & $-42^{\circ}$ & & & & & & & & \\
\hline 9 & 23.3 & 252 & 25 & 1 & & 2 & 3.7 & & & \\
\hline 37 & 2.0 & 289 & 5 & 2 & & 2 & 1.2 & & & \\
\hline 48 & 3.4 & 180 & 35 & 1 & & 9 & 4.8 & 20.4 & 0.29 & 55 \\
\hline 182 & 21.5 & 205 & 6 & 4 & & 18 & 2.5 & 34.0 & 0.37 & 30 \\
\hline 222 & 0.2 & 255 & 2 & 2 & & 4 & 3.2 & & & \\
\hline Slice & $\delta=$ & $-45^{\circ}$ & & & & & & & & \\
\hline 48 & 3.4 & 190 & 35 & 4 & 2 & 14 & 4.3 & 25.5 & 0.47 & 63 \\
\hline 182 & 21.6 & 188 & 6 & 1 & & 5 & 2.1 & & & \\
\hline 183 & 21.2 & 270 & 2 & 2 & & 9 & 2.5 & 25.1 & 0.29 & 2 \\
\hline 197 & 22.6 & 252 & 11 & 2 & & 6 & 2.3 & 14.4 & 0.26 & 64 \\
\hline 206 & 22.9 & 342 & 4 & 1 & & 4 & 3.6 & & & \\
\hline $\operatorname{slg} 3$ & 1.3 & 250 & & & & 8 & 3.0 & 9.35 & 0.67 & 79 \\
\hline
\end{tabular}

The columns in the table are as follows:

Column (1): The supercluster number from the catalogue of superclusters of Abell clusters by Einasto et al. (2001).

Columns (2): The RA of the supercluster (hours).

Columns (3): The distance to the supercluster (in $h^{-1} \mathrm{Mpc}$ ).

Columns (4): The number of Abell clusters in the supercluster.

Column (5): The number of Abell clusters in the part of supercluster inside the LCRS slice.

Column (6): The number of X-ray clusters among Abell clusters in this part of the supercluster that is inside LCRS slice.

Column (7): The number of LCLGs in the supercluster region.

Column (8): The maximum relative density in the supercluster region, $\Delta$, in units of the mean density.

Column (9): The length of the largest semiaxis $a$ of the ellipsoid of concentration of LCLG superclusters (in $h^{-1} \mathrm{Mpc}$ ).

Column (10): The ratio of semiaxis $b / a$ of the ellipsoid of concentration.

Column (11): The angles between line-of-sight and the large semiaxis $a$ of LCLG superclusters (in degrees). 


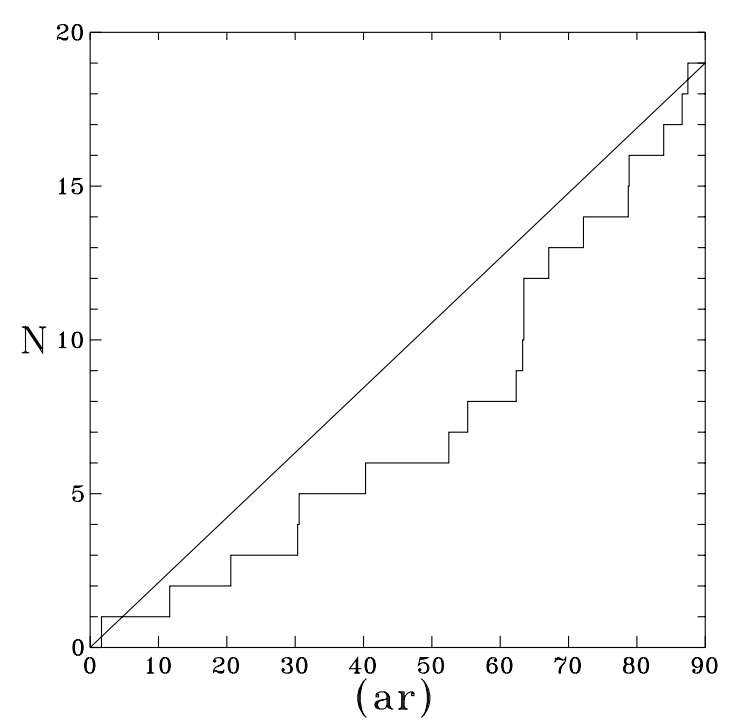

Fig. 2. The angle between the line-of-sight and the large semiaxes of the LCLG superclusters (Table 1). The diagonal corresponds to the uniform distribution.

In the case of contemporary deep surveys as the LCRS, where the galaxies with measured redshifts cover only a narrow slice, the shape of the ellipsoid strongly depends on the parameters of the survey. For a thin layer it is possible to use a 2D approximation, ignoring the third coordinate (in our case, the declination). Since the geometry of slices is far from a plane-parallel sheet we shall use the 3D algorithm to approximate the shapes of systems. In this way we get 3D objects that correspond to the 3D "slices" cut from the larger 3D superclusters (Abell superclusters).

In Table 1 we present some parameters of the ellipsoid of concentration for all systems composed of at least 6 LCLGs. In Fig. 2 we plot the distribution of angles between the lineof-sight and the large semiaxes of the LCLG superclusters (Table 1). The diagonal corresponds to a uniform distribution. The systems are moderately elongated (the mean axes ratio $3: 1$ ) and show a tendency to be oriented perpendicularly to the line of sight. The same tendency has been found for Abell superclusters (Jaaniste et al. 1998).

\subsection{LCLGs and Abell superclusters}

Here we describe shortly the most prominent Abell superclusters crossed by the LCRS slices.

The most prominent Abell supercluster crossed by the Northern LCRS slices is the supercluster SCL126 in the direction of the Virgo constellation (Fig. 3). Four Abell clusters of total seven member clusters of this supercluster are located in the Las Campanas slice $\delta=-3^{\circ}$ within a sphere of a diameter of about $10 \mathrm{~h}^{-1} \mathrm{Mpc}$. Three of these four clusters are strong $\mathrm{X}$-ray sources. The fifth X-ray cluster in this supercluster is Abell 1750, but it is located outside the slice. This cluster is a merging binary cluster (Donelly et al. 2001). Four Abell clusters in this supercluster are radio sources. Such a concentration of rich optical, X-ray, and radio clusters in one supercluster in a very small volume makes SCL126 one of the most unusual

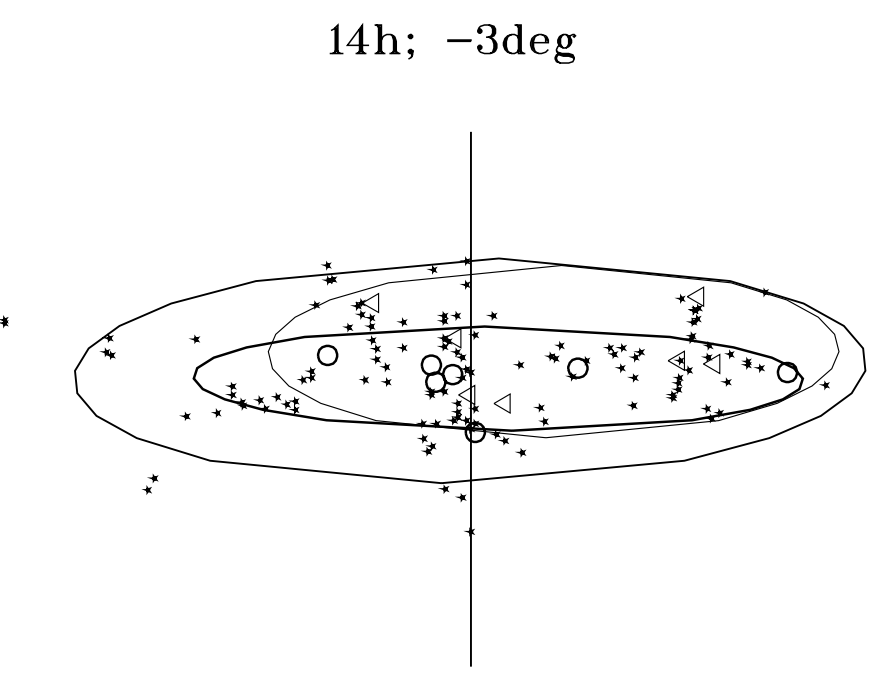

\section{Observer}

Fig. 3. The distribution of Abell clusters (open circles), Las Campanas Loose Groups (triangles) and LCRS galaxies (stars) in the region of the supercluster SCL126 along line-of-sight, with shape ellipses. Open circles denote Abell clusters, triangles - LCLGs, stars - LCRS galaxies.

superclusters currently known. There are three LCLGs in the central area of the supercluster. All these groups are unusually rich. Table 1 shows that the local density in the area of this supercluster is the largest in the whole survey, $\Delta>5$.

Another rich supercluster in this slice is the supercluster SCL100 with 9 member Abell clusters (the supercluster Leo A). The members of this supercluster are very close to the LCRS slices being located almost all in one plane. Three member clusters lie in the slice $\delta=-3^{\circ}$, and one in the slice $\delta=-6^{\circ}$. Altogether there are 26 loose groups from the LCRS in this supercluster. However, the properties of this supercluster differ from those of SCL126. There are no X-ray clusters among these clusters. One cluster, Abell 1200, is a radio source. The distances between the Abell clusters in this supercluster are quite large, and this supercluster resembles rather a filament of clusters. Table 1 shows that this filament-like supercluster is located almost along the line-of-sight.

A part of the Sextans supercluster (SCL 88) is seen in all three Northern slices. The Abell cluster members of the Sextans supercluster closest to the LCRS slices are A978 (the slice $\delta=-6^{\circ}$ ), and A970 (the slice $\delta=-12^{\circ}$, this is an X-ray cluster). In the slice $\delta=-3^{\circ} 23$ groups form a system that is an extension of this supercluster. The groups themselves in this extension are relatively poor - the richest loose group here has $N_{\text {ACO }}=29$, being poorer than Abell clusters of richness class $R=0$.

Another supercluster, seen in the slice $\delta=-12^{\circ}$, is the supercluster SCL119. Its member cluster Abell 1606 is an X-ray source and is associated with three loose groups.

The most prominent supercluster crossed by all Southern LCRS slices (and one of the richest superclusters known) is the Horologium-Reticulum supercluster (SCL48), 47 LCLGs being associated with this supercluster (Fig. 4). This supercluster contains two X-ray clusters and a number of APM clusters 

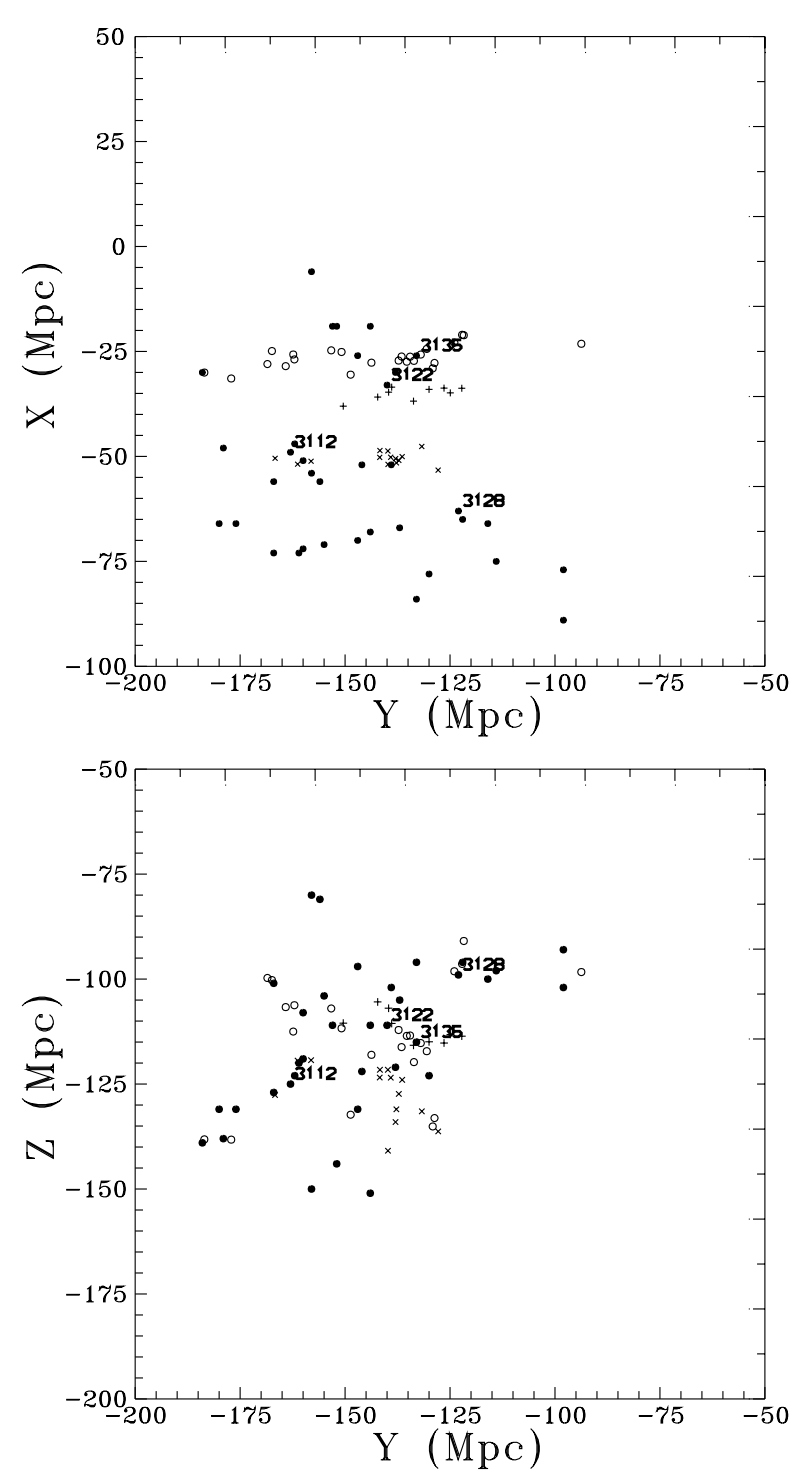

Fig. 4. The distribution of Abell clusters and Las Campanas loose groups in the region of the supercluster SCL48 (the HorologiumReticulum supercluster) in supergalactic $Y X$ (upper panel) and $Y Z$ (lower panel) coordinates in Mpc. Filled circles denote Abell clusters, open circles - LCLGs from the slice $\delta=-39^{\circ}$, crosses - LCLGs from the slice $\delta=-42^{\circ}$, and $\times$ 's - LCLGs from the slice $\delta=-45^{\circ}$. The Abell numbers of the richest Abell clusters in this supercluster are given (see text).

(Einasto et al. 2002b). One concentration of Abell clusters and LCLGs in this supercluster is centred on the very rich Abell cluster A3135 (the richness class $R=2$, the slice $\delta=-39^{\circ}$ ) that is associated with 7 loose groups. Another concentration of clusters in the Horologium-Reticulum supercluster is crossed by the slice $\delta=-45^{\circ}$. The richest Abell cluster in this region is Abell 3112, an X-ray and radio source. However, the richest concentration of LCLGs in this slice is located around another member cluster of this supercluster, Abell 3133 (the richness class $R=0$ ). A third concentration of groups and clusters in this supercluster is located around the Abell cluster 3128 (Rose et al. 2002) at the distance of about $40 \mathrm{~h}^{-1} \mathrm{Mpc}$ from the cluster Abell 3135. This concentration, however, lies outside the boundaries of the LCRS slices. All these concentrations are connected by filaments of galaxies, groups and clusters that surround underdense regions (see also Rose et al. 2002).

Another very rich supercluster crossed by the LCRS slices is the Sculptor supercluster (SCL9). There are five Abell clusters in the region of the LCRS slices from this supercluster, 4 in the slice $\delta=-39^{\circ}$ and 1 in the slice $\delta=-42^{\circ}$. Altogether there are 16 loose groups near these rich clusters in this supercluster.

The supercluster SCL23 in the slice $\delta=-39^{\circ}$ consists of two Abell clusters, Abell 2860 (a radio source) and Abell 2911. There are 11 LCLGs in this region. This supercluster is seen in the ESP survey as a very strong density enhancement in the galaxy distribution (Vettolani et al. 1997). This supercluster separates two voids, each of which have diameters of about $100 h^{-1} \mathrm{Mpc}$. On opposite sides of these voids are the Horologium-Reticulum and the Sculptor superclusters.

The Southern slices $\delta=-42^{\circ}$ and $\delta=-45^{\circ}$ cross the supercluster SCL182. All 6 member Abell clusters of this supercluster are located in these slices. The cluster A3809 is an X-ray source.

\subsection{Rich systems of loose groups only}

Rich systems (superclusters) of LCLGs (Table 1) contain at least 8 LCLGs for the linking length $6 h^{-1} \mathrm{Mpc}$ and have no Abell clusters.

One system consisting of 8 loose groups is located in the slice $\delta=-3^{\circ}$ in the void separating the Abell superclusters SCL 88 (Sextans), 126, and 155.

Another such high-density system of loose groups is located in the slice $\delta=-12^{\circ}$ at a distance of about $220 h^{-1} \mathrm{Mpc}$. The closest Abell cluster to this system is Abell 1317, that lies at a distance of about $15 \mathrm{~h}^{-1} \mathrm{Mpc}$ from the richest group in this system, LCLG-12 092. This supercluster has a "spider-like" appearance.

In the slice $\delta=-45^{\circ}$ there is a system of 8 loose groups located between voids at a distance of about $250 \mathrm{~h}^{-1} \mathrm{Mpc}$. An about $100 h^{-1} \mathrm{Mpc}$ void separates this system from the Horologium-Reticulum supercluster. There are some galaxy systems in this void, but no rich clusters or superclusters. This system resembles the Great Wall, a rich filament of galaxies and groups of galaxies connecting superclusters.

The existence of supercluster systems that contain only LCLGs and separate huge voids of diameter of about $100 h^{-1} \mathrm{Mpc}$ agrees with the earlier findings by Einasto et al. (1997) and Frisch et al. (1995). These earlier studies found that huge voids in the supercluster-void network are of similar size, about $100 h^{-1} \mathrm{Mpc}$, but the properties of void walls range from those of poor superclusters to very rich superclusters containing tens of rich (Abell) clusters.

\section{Properties of LCLGs in different systems}

Let us now compare the properties of LCLGs in Abell superclusters with the properties of LCLGs in rich systems of LCLGs containing no Abell clusters (LCLG.scl and LCLG.slg, respectively; the sample LCLG.slg includes also the outer members of SCL88 in the slice $\delta=-3^{\circ}$ ). 
Table 2. Median and upper quartile (in parentheses) values of LCLG properties.

\begin{tabular}{lrrrrrr}
\hline \hline Sample & $N_{\text {group }}$ & $N_{\text {obs }}$ & $N_{\text {ACO }}$ & $\begin{array}{r}\sigma_{\text {los }} \\
\mathrm{km} \mathrm{s}^{-1}\end{array}$ & $\begin{array}{r}\log M_{\text {vir }} \\
h^{-1} M_{\odot} \\
(6)\end{array}$ & $\begin{array}{r}\log L_{\text {tot }} \\
h^{-2} L_{\odot} \\
(1)\end{array}$ \\
& $(2)$ & $(3)$ & $(4)$ & $(5)$ & $15)$ \\
\hline LCLG.scl & 204 & $4.5(8.0)$ & $15.5(25.0)$ & $215(290)$ & $13.45(14.05)$ & $11.15(11.65)$ \\
LCLG.slg & 47 & $4.5(7.5)$ & $9.5(15.2)$ & $175(280)$ & $13.25(13.70)$ & $10.95(11.20)$ \\
& & & & & & \\
LCLG.all & 1495 & $4.0(5.5)$ & $16(25.0)$ & $164(270)$ & $13.20(13.75)$ & $11.10(11.45)$ \\
\hline
\end{tabular}

The columns are as follows:

Column (1): Sample identification (see text).

Column (2): $N_{\text {group }}$, the number of LCLGs in the sample.

Column (3): $N_{\text {obs }}$, the observed number of LCRS galaxies in groups.

Column (4): $N_{\mathrm{ACO}}$, the group Abell counts.

Column (5): $\sigma_{\text {los }}$, the group rms line-of-sight velocities (in units of $\mathrm{km} \mathrm{s}^{-1}$ ).

Column (6): $M_{\text {vir }}$, the virial mass of a group (in units of $h^{-1} M_{\odot}$ ).

Column (7): $L_{\mathrm{tot}}$, the total group luminosity in the LCRS $R$-band (in units of solar luminosity $\left(h^{-2} L_{\odot}\right)$ ).

Several physical properties have been calculated for each group in the LCLG catalogue (TUC). These include the observed number of group member galaxies $N_{\mathrm{obs}}$, the line-of-sight velocity rms $\sigma_{\text {los }}$, the virial mass $M_{\text {vir }}$, the total luminosity $L_{\text {tot }}$, and the Abell counts $N_{\mathrm{ACO}}$. We refer to TUC for details of how these properties were estimated. In Table 2 we give the values of these properties for loose groups from different systems, as well as for the total sample of LCLGs.

Two measures of a group's richness are its observed number of galaxies, $N_{\mathrm{obs}}$, and its Abell count, $N_{\mathrm{ACO}}$, calculated taking into account the selection effects (see TUC). We see that if we use these estimated Abell counts as a measure of the group richness, loose groups in Abell superclusters tend to be richer than loose groups in systems without Abell clusters. Only the two richest groups in the sample of systems of loose groups, LCRS.slg, have the Abell counts larger than 30 - this population consists of intrinsically poor loose groups. In contrast, in the population of loose groups in Abell superclusters the mean Abell count $N_{\mathrm{ACO}}=20$ and more than $15 \%$ of loose groups have $N_{\mathrm{ACO}}$ larger than 30 . The richest group in this sample has the Abell count $N_{\mathrm{ACO}}=120$, equivalent to a richness class $R=2$ cluster.

Additionally, let us take as an example the Abell supercluster SCL222 that consists of two Abell clusters and is probably completely embedded within the LCRS slice $\delta=-42^{\circ}$. In this supercluster alone there are 4 loose groups, and three of them are richer than $N_{\mathrm{ACO}}=30$ - richer than any group from the sample of loose groups from systems without Abell clusters. This may indicate that, although the local density of groups is rather high in the systems without Abell clusters, all loose groups in these systems are intrinsically poor. This is a hint that the absence of Abell clusters in these systems is due to the poorness of individual groups in these region and not due to possible incompleteness in the Abell catalogue.

The Kolmogorov-Smirnov test shows that the differences in the distribution of group richnesses between the two samples are statistically significant at the $90 \%$ confidence level.

The rms line-of-sight velocity of loose groups, $\sigma_{\text {los }}$, in the Abell superclusters is about 1.2 times larger than that of loose groups from systems without Abell clusters (LCRS.slg). There are no loose groups in this population with the rms velocity larger than $\sigma_{\text {los }}=425 \mathrm{~km} \mathrm{~s}^{-1}$. The Kolmogorov-Smirnov test shows that the differences in the distribution of group rms velocities between the two group samples are statistically significant at the $99 \%$ confidence level.

Comparison of virial masses of loose groups, $M_{\mathrm{vir}}$, shows that loose groups in Abell superclusters have masses that are about 1.6 times larger than masses of loose groups in systems without Abell clusters. The Kolmogorov-Smirnov test shows that the differences in the distribution of group virial masses between the two samples are statistically significant at the $75 \%$ confidence level.

The total luminosities of groups, $L_{\text {tot }}$, show that loose groups in Abell superclusters are about 1.6 times more luminous than loose groups in systems without Abell clusters. The Kolmogorov-Smirnov test shows that the differences in the distribution of group luminosities between the two samples of groups are statistically significant at the $95 \%$ confidence level.

In order to study how far the environmental enhancement of loose group properties extends, we calculated for loose groups in Abell superclusters the distance to the nearest Abell cluster. In Fig. 5 we plot the rms line-of-sight velocities of loose groups in superclusters against the distance to the nearest Abell cluster in a supercluster. This figure shows a decrease in the velocity dispersions of loose groups with an increase in the distance to the nearest Abell cluster. Enhancement of properties of loose groups extends quite far from Abell clusters, up to about 15-20 $h^{-1} \mathrm{Mpc}$. At distances larger than approximately $20 \mathrm{~h}^{-1} \mathrm{Mpc}$ (in our sample these loose groups are distant members of the supercluster SCL 48, the Horologium-Reticulum supercluster), this phenomenon becomes weaker.

The loose groups from an outer population of the supercluster SCL88 in the slice $\delta=-3^{\circ}$ lie at distances of about $16 h^{-1} \mathrm{Mpc}$ from the nearest Abell cluster in this supercluster (this Abell cluster is seen in the LCRS slice $\delta=-6^{\circ}$ ). The nearest Abell cluster to the loose groups in the LCRS.slg1 system is located at a distance of about $30 \mathrm{~h}^{-1} \mathrm{Mpc}$. Likewise, the nearest Abell clusters to the loose groups in the LCRS.slg2 and LCRS.slg3 systems are at distances of about $15 h^{-1} \mathrm{Mpc}$ and about $35 h^{-1} \mathrm{Mpc}$, respectively. Thus, groups not belonging to 


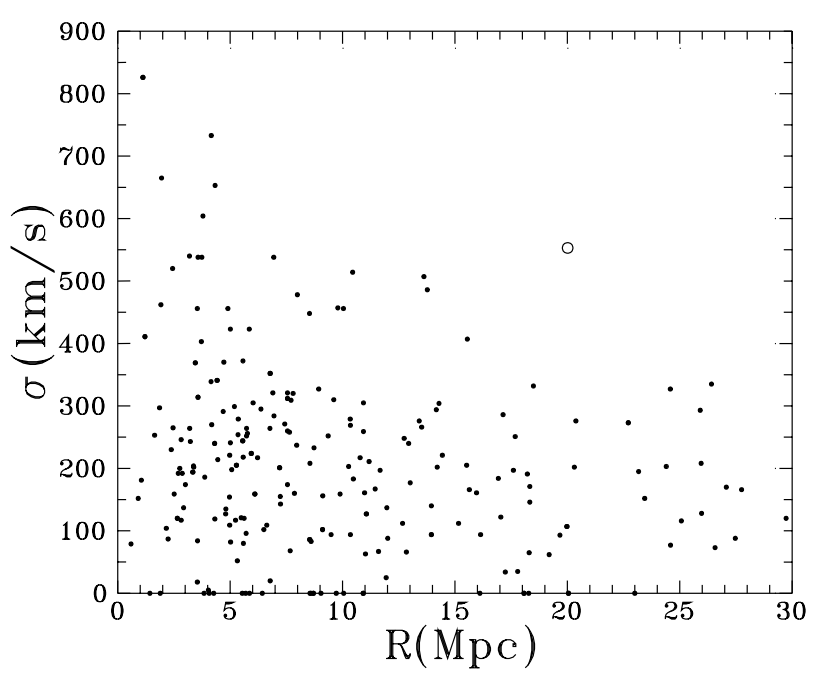

Fig. 5. The rms line-of-sight velocities of loose groups in Abell superclusters against the distances from the nearest Abell cluster in a supercluster. The open circle represents LCLG-12 163, that is located at an end of a filament centred on an Abell cluster from SCL119.

superclusters or those groups which are outer members of superclusters do not show environmentally enhanced properties as do the inner members of superclusters.

To summarise, these results extend the environmental enhancement of mass, velocity dispersion, and luminosity of loose groups in the vicinity of rich clusters of galaxies found by Einasto et al. (2003a) to the loose groups within Abell superclusters. This effect is absent in systems which contain no rich clusters. We found indications that this effect is also absent in the case of loose groups from outer parts of superclusters.

\section{LCLGs and the supercluster-void network}

The large scale distribution of Abell clusters and superclusters was described in Einasto et al. (1997). In particular, it was shown that $75 \%$ of very rich superclusters are located in the so-called Dominant Supercluster Plane (DSP) that crosses the Local Supercluster Plane at almost right angles and consists of chains of superclusters and voids between them. Let us now study the distribution of LCLGs with respect to Abell superclusters and the Dominant Supercluster Plane.

Figure 6 shows the distribution of Abell clusters and the location of the LCRS slices with respect to the superclustervoid network. The Las Campanas slices cross several rich superclusters in the Dominant Supercluster Plane: the Sculptor supercluster and the Horologium-Reticulum supercluster in the Southern sky, and the Leo A supercluster in the Northern sky. The Southern slice at $\delta=-39^{\circ}$ goes almost through the DSP. Of the Northern slices, the slice at $\delta=-3^{\circ}$ is closest to the DSP; the other Northern slices cross the voids between superclusters. The Northern slice at $\delta=-6^{\circ}$ crosses the region most devoid of galaxies and galaxy systems. This may be one of the reasons why the number of LCLGs in this slice is much smaller than the number of groups in other slices.

\section{Discussion}

\subsection{Selection effects}

The LCRS observations were performed in a fixed apparent magnitude interval and therefore galaxies fainter or brighter than the survey limits are not included in the survey. Thus groups consisting of faint galaxies can be detected only in the nearest regions of the survey. With increasing distance, groups containing fainter galaxies gradually disappear from the sample. This effect was discussed in detail in Einasto et al. (2003a) and in Heinämäki et al. (2003). In Fig. 7 we show the distancedependent selection effect for loose groups from different systems. Figure 7 shows that this distance-dependent selection effect affects loose groups from different systems in a similar way.

In addition, in Fig. 8 we plot the maximum relative supercluster densities from Table 1 against the distances of superclusters. This figure shows that there is no distance-dependent bias for supercluster densities. This means that the selection effects have been taken into account properly when determining the density field superclusters.

Einasto et al. (2003a) and Heinämäki et al. (2003) discussed several selection effects that could affect the properties of loose groups. We analysed the properties of loose groups in high density regions around rich clusters and showed that selection effects cannot artificially enhance the properties of loose groups in high density regions.

In Einasto et al. (2003a), Einasto et al. (2003b) and Einasto et al. (2003c) we discuss also several other distance-dependent selection effects. Our analysis of properties of groups in a wide range of environments (not just in high density environment of superclusters) shows that groups of lower luminosity tend to be located in lower density environment (as shown earlier by Lindner et al. 1995) at all distances.

\subsection{Sizes and orientations of superclusters}

Table 1 shows that the lengths of the largest semiaxes of superclusters as determined by LCLGs are of order of $25 \mathrm{~h}^{-1} \mathrm{Mpc}$. This value is close to the minor semiaxes of Abell superclusters determined using triaxial ellipsoids (Jaaniste et al. 1998). This estimate of the sizes of superclusters is in accordance with the scales found in the power spectrum of galaxies. At scales of about $30-60 \mathrm{~h}^{-1} \mathrm{Mpc}$ the power spectrum of galaxies from deep surveys indicate a possible presence of a "wiggle" - an excess near these scales and a minimum at larger scales (Silberman et al. 2001; Percival et al. 2001). Gramann \& Hütsi (2001) interpreted this scale as a scale that corresponds to a size of a typical supercluster (Jaaniste et al. 1998).

The orientation of superclusters with respect to the line-ofsight has an excess of systems oriented perpendicular to the line of sight. In Jaaniste et al. (2003) we found a similar tendency of orientations in the case of superclusters of LCLGs determined using the FoF method and a neighbourhood radius $R=12 h^{-1} \mathrm{Mpc}$. In a recent study of the $2 \mathrm{dF}$ survey Peacock et al. (2001) found evidence of recessional velocities caused by a systematic infall of galaxies into superclusters. Our results on 

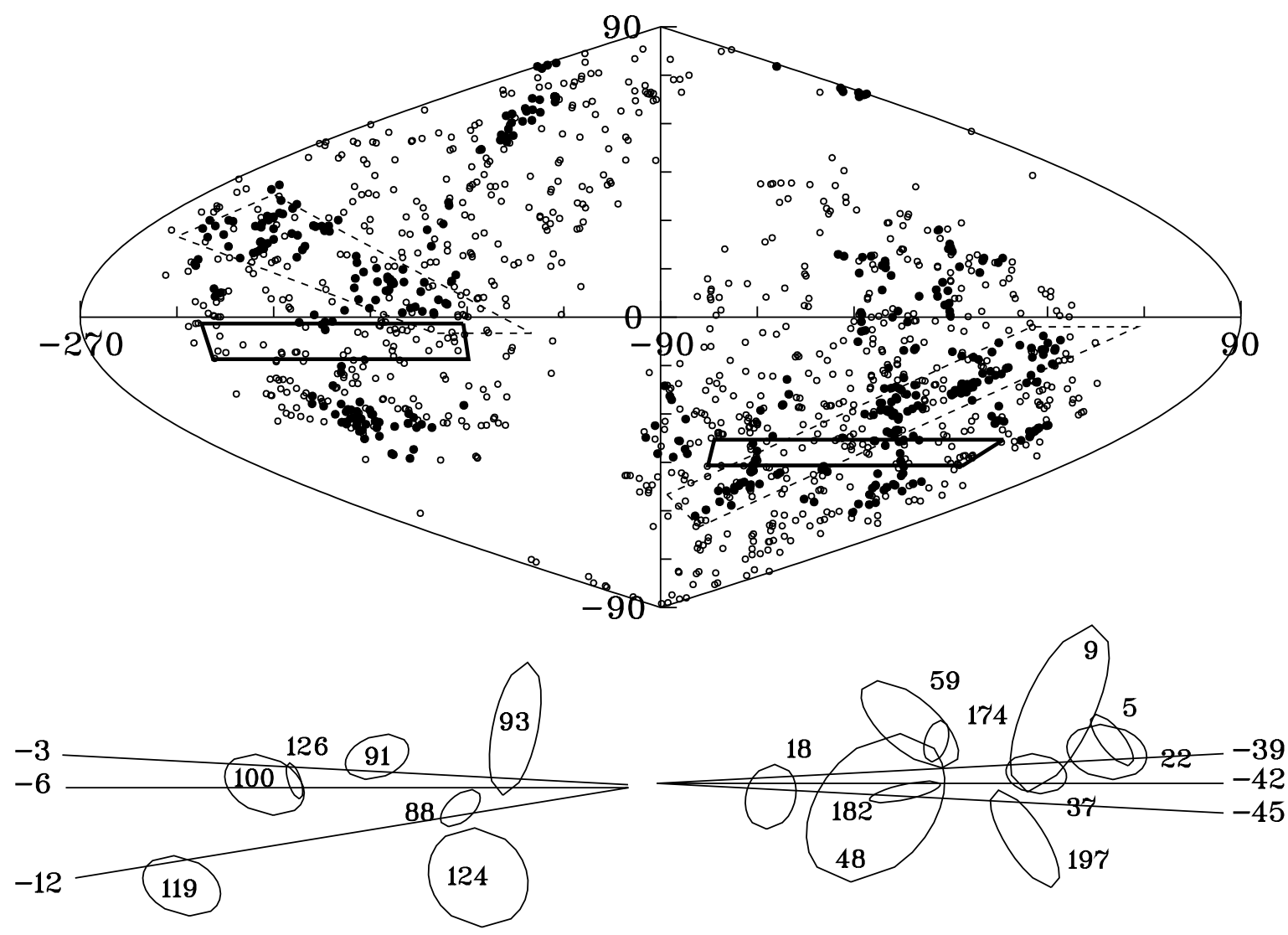

Fig. 6. Upper panel: the Abell clusters in equatorial coordinates. Filled circles show the Abell clusters located in superclusters of richness 8 and more members, open circles mark the Abell clusters in less rich superclusters. Solid lines show the location of the LCRS slices, dashed lines the location of the Dominant Supercluster Plane. Lower panels show intersections of supercluster ellipsoids with the LCRS slices

the orientations of superclusters may be evidence of the same effect.

Moreover, the supercluster SCL126 in the direction of the Virgo constellation can be interpreted as an example of such infall. In this case the ellipsoids calculated using data on Abell clusters, loose groups and individual galaxies were shown above (Fig. 3). In all cases the ellipsoid with the axes ratio about 1:4 is located perpendicularly to the line of sight. Jaaniste et al. (1998) found that this is one of the flattest and thinnest superclusters, being located almost perpendicularly with respect to the line of sight. This may be an evidence of the "squashing effect" of infalling galaxies into superclusters before turnaround or beginning of the relaxation (Kaiser 1987), accompanied by merging and other processes that cause X-ray and radio radiation from clusters in this supercluster (Sect. 4).

\subsection{Individual superclusters}

The LCRS slices are, in general, too thin to contain completely a whole supercluster, as traced by Abell clusters. However, the core region of the supercluster SCL126 is located in the slice $\delta=-3^{\circ}$. In this region the concentration of Abell and $\mathrm{X}$-ray clusters and Las Campanas loose groups and galaxies in very high. Superclusters like SCL126 may be detected though the Sunyaev-Zeldovich effect in surveys like the forthcoming Planck mission.

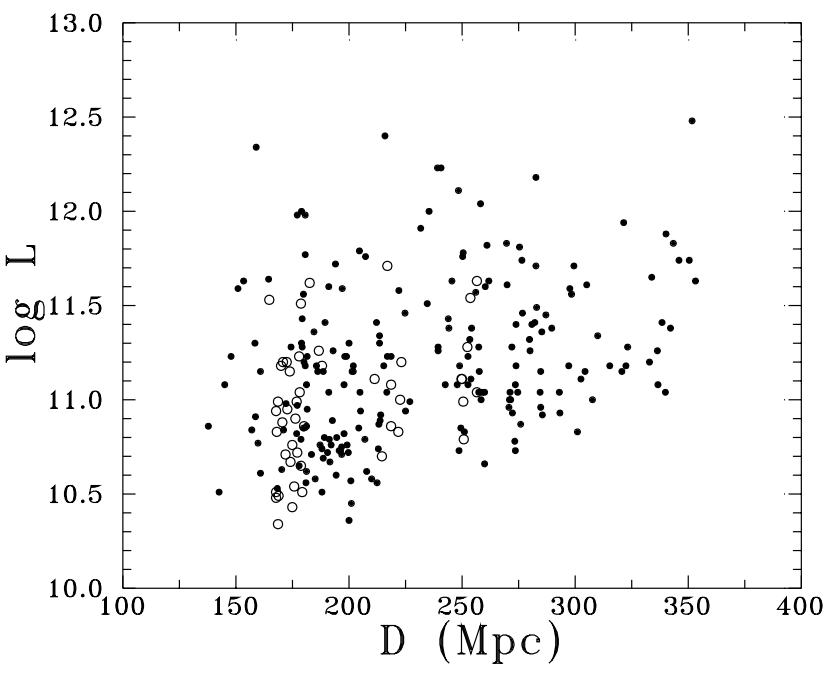

Fig. 7. Luminosities of LCRS loose groups from superclusters of Abell clusters (filled circles) and from systems without Abell clusters (open circles) (in units of solar luminosity $h^{-2} L_{\odot}$ ) versus the distances of the groups (in $h^{-1} \mathrm{Mpc}$ ).

Such a high concentration of clusters has been observed so far only in a very few superclusters. Among them are the Shapley supercluster (Bardelli et al. 2000) and the Aquarius supercluster (Caretta et al. 2002). A very small number of such a high density cores of superclusters is consistent with the 


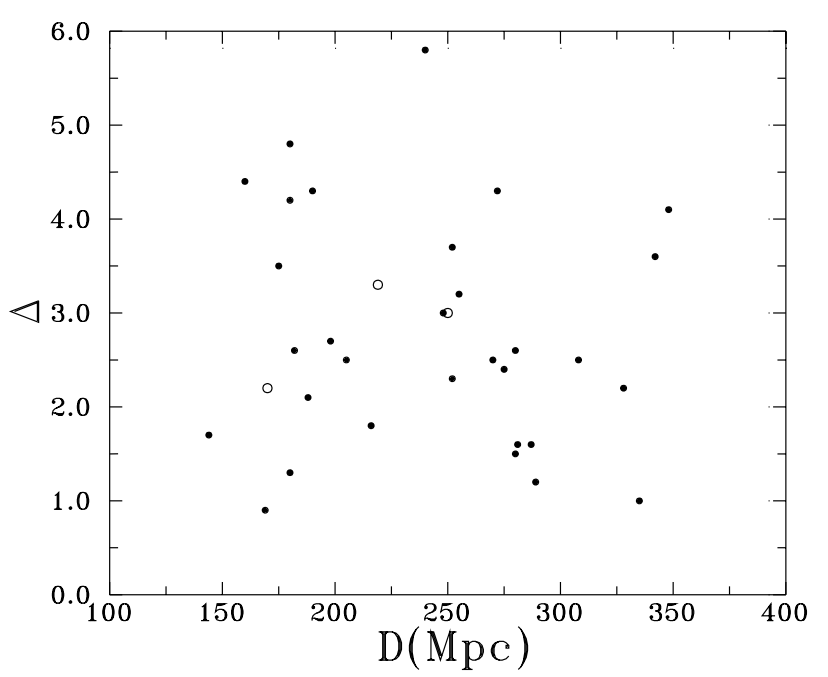

Fig. 8. Maximum relative densities in superclusters (Table 1) versus the distance of a supercluster. Filled circles correspond to superclusters of Abell clusters, open circles - to systems without Abell clusters.

results from $N$-body calculations which show that such high density regions (the cores of superclusters that may have started the collapse) are rare (Gramann \& Suhhonenko 2002).

In the case of the Horologium-Reticulum supercluster (SCL48) the LCRS data trace rather well two of the three concentrations of galaxies determined in this supercluster, using data on rich clusters (see also Rose et al. 2002).

The supercluster SCL100 is also located almost inside the slice $\delta=-3^{\circ}$. However, in contrast to SCL126, the local density in the region of this supercluster is not high. With its elongated shape this supercluster resembles a filament. Such long filament-like superclusters have also been detected in $N$-body calculations (Faltenbacher et al. 2002).

\subsection{Large scale structure}

In Jaaniste et al. (2003) we compared the smoothed density field of the Las Campanas Redshift survey calculated by Einasto et al. (2003c) using data on galaxies, with intersections of supercluster ellipsoids in the same regions. The intersection ellipses coincided well with the densest regions of the density field. In other words, the superclusters can be well traced using both the LCRS data and the Abell cluster data.

We note that the LCRS slices cross the supercluster-void network at such an angle that the $120 \mathrm{~h}^{-1} \mathrm{Mpc}$ scale that characterises the distribution of rich clusters and superclusters (Einasto et al. 1994) is not clearly expressed, and we see a smaller scale (of about $100 \mathrm{~h}^{-1} \mathrm{Mpc}$ ) as an excess in the correlation function of LCRS (Tucker et al. 1997) instead.

\subsection{Properties of groups in superclusters}

We showed that loose groups in Abell superclusters are more massive and luminous and have larger velocity dispersions than loose groups in systems without rich clusters. Our study extends the environmental enhancement of the mass and richness of loose groups in the vicinity of rich clusters of galaxies described in Einasto et al. (2003a) to larger scales up to about $15-20 h^{-1} \mathrm{Mpc}$ from rich clusters in superclusters.

These results are in accordance with those by Einasto et al. (2003b) who used a larger sample of groups and clusters from the Sloan survey to show that groups and clusters in high density environments have higher luminosities than those in low density environments.

Our results describe one aspect of the hierarchy of systems in the Universe, earlier characterised using the sizes of voids determined by different objects (Lindner et al. 1995; Arbabi-Bidgoli \& Müller 2002).

Several recent studies of the correlation function of nearby groups of galaxies show that properties of groups of galaxies in high density regions are different from properties of groups on average (Giuricin et al. 2001; Girardi et al. 2000; Merchan et al. 2000). Stronger clustering is an indication that these groups could be located in the high density regions of superclusters (Einasto et al. 1997; Tago et al. 2002).

Additionally, several studies of clusters of galaxies have provided evidence that properties of rich clusters depend on their large scale environment (Einasto et al. 2001; Plionis \& Basilakos 2002; Schuecker et al. 2001; Chambers et al. 2002; Novikov et al. 1999) up to a distance of about $20 \mathrm{~h}^{-1} \mathrm{Mpc}$. This distance is close to the so-called "pancake scale" (Melott \& Shandarin 1993), and corresponds to the mean thickness of superclusters (Einasto et al. 1994, 1997; Jaaniste et al. 1998). This distance is also close to that up to which the environmental enhancement of loose groups hase been detected in the present study.

Suhhonenko (2002) has demonstrated using different $N$-body simulations that in simulated superclusters more massive clusters are located in the central regions of superclusters.

Gottlöber et al. (2002) and Faltenbacher et al. (2002) analysed high-resolution simulations of formation of galaxies, groups, and clusters and found a significant enhancement of the mass of haloes in the environment of other haloes. This effect is especially significant at scales below $10 \mathrm{~h}^{-1} \mathrm{Mpc}$. Therefore, environmental enhancement of the halo mass is a direct evidence for the process of the hierarchical formation of galaxy and cluster haloes.

\section{Conclusions}

We studied the Las Campanas loose groups in superclusters of Abell clusters. We described the superclusters that are crossed by LCRS slices, and the large-scale distribution of the LCLGs in the supercluster-void network.

Our results show that the orientation of superclusters, as determined by LCLGs, has an excess of systems oriented perpendicularly to the line-of-sight. The Las Campanas loose groups in superclusters are richer and more massive than loose groups in systems that do not belong to superclusters. The data about galaxies, loose groups and rich clusters show that the supercluster SCL126 has a very high density core containing several $\mathrm{X}$-ray clusters. This supercluster is located almost perpendicularly in respect to the line-of-sight. We assume that this may be due to infall of galaxies into the supercluster. 
Our study indicates the importance of the role of superclusters as high density environment which affects the properties (formation and evolution) of galaxy systems.

Acknowledgements. We thank Erik Tago and Heinz Andernach for providing us with the compilation of the data on Abell clusters. We thank Enn Saar and Sahar Allam for stimulating discussions. The present study was supported by the Estonian Science Foundation grant 4695 and by the Estonian Research and Development Council grant TO 0060058S98. P.H. was supported by the Finnish Academy of Sciences (grant 46733). D.L.T. was supported by the US Department of Energy under contract No. DE-AC02-76CH03000. This study has made use of the NASA/IPAC Extragalactic Database (NED) which is operated by the Jet Propulsion Laboratory, Caltech, under agreement with the National Aeronautics and Space Association.

\section{References}

Abell, G. 1958, ApJS, 3, 211

Abell, G., Corwin, H., \& Olowin, R. 1989, ApJS, 70, 1

Andernach, H., \& Tago, E. 1998, in Large Scale Structure: Tracks and Traces, ed. V. Müller, S. Gottlöber, J. P. Mücket, \& J. Wambsganss (Singapore: World Scientific), 147

Arbabi-Bidgoli, S., \& Müller, V. 2002, MNRAS, 332, 205

Bardelli, S., Zucca, E., Zamorani, G., Moscardini, L., \& Scaramella, R. 2000, MNRAS, 312, 540

Basilakos, S., Plionis, M., \& Rowan-Robinson, M. 2001, MNRAS, 323,47

Caretta, C. A., Maia, A. G., Kawasaki, W., \& Willmer, C. N. A. 2002, AJ, 123, 1200

Chambers, S. W., Melott, A. L., \& Miller, C. J. 2002, ApJ, 565, 849

Colless, M., Dalton, G., Maddox, S., et al. 2001, MNRAS, 328, 1039

De Grandi, S., Böhringer, H., Guzzo, L., et al. 1999, ApJ, 514, 148

Donelly, R. H., Forman, W., Jones, C., et al. 2001, ApJ, 562, 254

Einasto, J., Einasto, M., Frisch, P., et al. 1997, MNRAS, 289, 801

Einasto, J., Hütsi, G., Einasto, M., et al. 2003b, A\&A, 405, 425

Einasto, J., Einasto, M., Hütsi, G., et al. 2003c, A\&A, submitted

Einasto, M., Einasto, J., Tago, E., Dalton, G., \& Andernach, H. 1994, MNRAS, 269, 301

Einasto, M., Tago, E., Jaaniste, J., Einasto, J., \& Andernach, H. 1997, A\&AS, 123, 119

Einasto, M., Einasto, J., Tago, E., Andernach, H., \& Dalton, G. 2001, $\mathrm{AJ}, 122,2222$

Einasto, M., Einasto, J., Tago, E., et al. 2002, AJ, 123, 51

Einasto, M., Einasto, J., Müller, V., Heinämäki, P., \& Tucker, D. L. 2003a, A\&A, 401, 851

Faltenbacher, A., Gottlöber, S., Kerscher, M., \& Müller, V. 2002, A\&A, 295, 1

Frisch, P., Einasto, J., Einasto, M., et al. 1995, A\&A, 296, 611

Girardi, M., Boschin, W., \& da Costa, L. N. 2000, A\&A, 353, 57

Giuricin, G., Samurovic, S., Girardi, M., Mezzetti, M., \& Marinoni, C. $2001, \mathrm{ApJ}, 554,857$
Gottlöber, S., Kerscher, M., Klypin, A., Kravtsov, A., \& Müller, V. 2002, A\&A, 387, 778

Gramann, M., \& Suhhonenko, I. 2002, MNRAS, 337, 1417

Gramann, M., \& Hütsi, G. 2001, MNRAS, 327, 538

Heinämäki, P., Saar, E., Einasto, J., Einasto, M., \& Tucker, D. 2003, A\&A, 397, 63

Jaaniste, J., Tago, E., Einasto, M., et al. 1998, A\&A, 336, 35

Jaaniste, J., Einasto, M., \& Einasto, J. 2003, Deep slices and the Supercluster-void Network, ed. D. Barbosa, JENAM 2002, in press

Jõeveer, M., \& Einasto, J. 1978, in The Large Scale Structure of the Universe, ed. M. S. Longair, \& J. Einasto (Dordrecht, Holland: Reidel), 241

Kolokotronis, V., Basilakos, S., \& Plionis, M. 2002, MNRAS, 331, 1020

Kaiser, N. 1987, MNRAS, 227, 1

Korn, G. A., \& Korn, T. M. 1961, Mathematical Handbook for Scientists and Engineers (McGraw-Hill Book Co., Inc.)

Kull, A., \& Böhringer, H. 1999, A\&A, 341, 23

Landy, S. D., Shectman, S. A., Lin, H., et al. 1996, ApJ, 456, L1

Ledlow, M. J., \& Owen, F. 1995, AJ, 109, 853

Lindner, U., Einasto, J., Einasto, M., et al. 1995, A\&A, 301, 329

Melott, A. L., \& Shandarin, S. F. 1993, ApJ, 410, 496

Merchan, M., Maia, M. A. G., \& Lambas, D. G. 2000, ApJ, 545, 26

Novikov, D. I., Melott, A. L., Wilhite, B. B., et al. 1999, MNRAS, 304, L5

Peacock, J. A., Cole, S., Norberg, P., Baugh, C. M., et al. 2001, Nature, 410,169

Percival, W. J., Baugh, C. M., Bland-Hawthorn, J., et al. 2001, MNRAS, 327, 1297

Plionis, M., \& Basilakos, S. 2002, MNRAS, 329, L47

Plionis, M., Valdarnini, R., \& Jing 1992, ApJ, 398, 12

Rines, K., Mahdavi, A., Geller, M. J., et al. 2001, ApJ, 555, 558

Rose, J. A., Gaba, A. E., Christiansen, W. A., et al. 2002, AJ, 123, 1216

Schuecker, P., Boehringer, H., Reiprich, T. H., \& Feretti, L. 2001, A\&A, 378, 408

Schwope, A. D., Hasinger, G., Lehmann, I., et al. 2000, Astron. Nachr., 321, 1 (RBS)

Shectman, S., Landy, S., Oemler, A., et al. 1996, ApJ, 470, 122

Silberman, L., Dekel, A., Eldar, A., \& Zehavi, I. 2001, ApJ, 557, 102

Suhhonenko, I. 2002, Ph.D. Thesis, Tartu University

Tago, E., Saar, E., Einasto, J., et al. 2002, AJ, 123, 37

Tarenghi, M., Tifft, W., Chincarini, G., Rood, H., \& Thompson, L. 1978, in The Large Scale Structure of the Universe, ed. M. S. Longair, \& J. Einasto (Dordrecht, Holland: Reidel), 241

Tucker, D. L., Oemler, A. Jr., Hashimoto, Y., et al. 2000, ApJS, 130, 237

Tucker, D. L., Oemler, A., Jr., Kirshner, R. P., et al. 1997, MNRAS, 285, L5

Vettolani, G., Zucca, E., Zamorani, G., et al. 1997, A\&A, 325, 954

West, M. 1989, ApJ, 347, 610

York, D. G., Adelman, J., Anderson, J. E., et al. 2000, AJ, 120, 1579

Zeldovich, Ya. B., Einasto, J., \& Shandarin, S. F. 1982, Nature, 300, 407 